\title{
ORQUIECTOMIA EM CAVALOS: COMPARAÇÃO ENTRE TRÊS TÉCNICAS EM RELAÇÃO AO TEMPO CIRÚRGICO, COMPLICAÇÕES PÓS-OPERATÓRIAS E TEMPO PARA ALTA HOSPITALAR
}

\author{
(Orchyectomy in horses: comparison between three techniques by surgical time, \\ post-operatory complications and medical release time)
}

Jéssica Rodrigues Silva-Meirelles*, Monalisa Lukascek Castro, Lilian Pamela Tapia Carreno Dornbusch, Rogério Luizari Guedes, Ivan Roque Barros-Filho, Peterson Triches Dornbusch

Programa de Pós-Graduação em Ciências Veterinárias. Setor de Ciências Agrárias. Universidade Federal do Paraná.

*Correspondência: jerodrigues_vet@yahoo.com.br

RESUMO: A orquiectomia é utilizada para esterilizar cavalos de baixo valor genético, melhorando o manejo. Pode ser realizada com anestesia geral ou local, em decúbito dorsal ou em estação. Algumas complicações podem ocorrer como hemorragia, edema, infecção e peritonite. O objetivo deste trabalho foi comparar o tempo cirúrgico, a ocorrência de complicações pós-operatórias e o tempo de internamento de cavalos submetidos à orquiectomia eletiva por meio de três diferentes técnicas empregadas por alunos e residentes do curso de medicina veterinária. Foram utilizados 25 cavalos, sendo separados em três grupos: grupo 1 (EMA, $n=8$ ), submetidos à orquiectomia bilateral em posição quadrupedal, bloqueio local e sedação, hemostasia com emasculador onde a túnica vaginal foi mantida aberta; grupo 2 (BRA, $n=8)$, submetidos à orquiectomia bilateral em posição quadrupedal, bloqueio local e sedação, hemostasia com duas abraçadeiras de náilon de 2,5mm e túnica vaginal mantida aberta; grupo 3 (ING, n=9), submetidos à orquiectomia bilateral em decúbito dorsal e anestesia geral, hemostasia com emasculador, e túnica vaginal e pele suturadas. $O$ tempo médio dos procedimentos foi de $16,3 \pm 2,5 \mathrm{~min}$ no grupo EMA, $14,5 \pm 1,6 \mathrm{~min}$ no grupo BRA e $64,4 \pm 16,6 \mathrm{~min}$ no grupo ING. No pós-operatório dos animais não foi observado sangramento no grupo ING; no grupo EMA houve sangramento grau 2 e no grupo BRA houve sangramento grau 1. O tempo médio de internamento foi de $22,7 \pm 3,2$ dias, $20,75 \pm 0,7$ dias e $10,7 \pm 4,5$ dias respectivamente. A orquiectomia bilateral sob efeito de anestesia geral e fechamento da túnica vaginal, demanda maior tempo cirúrgico quando comparada às orquiectomias realizadas com os cavalos em posição quadrupedal e manutenção da túnica aberta. Porém, não foram observadas complicações pósoperatórias nos animais castrados com essa técnica, diminuindo assim o tempo de internação hospitalar e os cuidados pós-operatórios.

Palavras-chave: castração; cirurgia; equinos

ABSTRACT: Orchiectomy is used to neuter low genetic value horses, improving management. Can be performed under general or local anesthesia, supine or quadrupedal position respectively. Some complications can occur such as bleeding, swelling, infection and peritonitis. The aim of this study was to compare the surgical time, the occurrence of postoperative complications and length of hospital care of horses undergoing elective orchiectomy through three different techniques used by veterinary school students and residents. 25 horses were selected, divided into three 
groups: group 1 (EMA, $n=8$ ) underwent bilateral orchiectomy in standing position, local anesthesia and sedation, hemostasis with emasculator and Tunica kept open; Group 2 (BRA, n=8), underwent bilateral orchiectomy in standing position, local anesthesia and sedation, hemostasis with two $2.5 \mathrm{~mm}$ nylon tiewraps and Tunica vaginalis kept open; Group 3 (ING, $n=9$ ), underwent bilateral orchiectomy in supine position and general anesthesia, hemostasis with emasculator, and closure of Tunica vaginalis and skin. The mean surgical times were $16.3 \pm 2.5 \mathrm{~min}$ in EMA, $14.5 \pm 1.6 \mathrm{~min}$ in ARB and $64.4 \pm 16.6 \mathrm{~min}$ in ING. There isn't postoperative bleeding in ING Group; EMA had a grade 2 bleeding and ARB had a grade 1 bleeding. The mean hospitalization time were $22.7 \pm 3.2$ days in EMA, 20.75 \pm 0.7 days in BRA and $10.7 \pm 4.5$ days in ING. Bilateral orchiectomy under general anesthesia and closure of the Tunica vaginalis, promotes longer surgical times when compared to orchiectomy in standing positions maintaining the surgical wounds opened. However, postoperative complications in the neutered animals weren't observed with this technique, thus reducing the length of hospital stay and postoperative care.

Key Words: neutering; surgery; equine

\section{INTRODUÇÃO}

A orquiectomia, também denominada emasculação ou castração, está entre os procedimentos mais realizados nos cavalos e é utilizada para esterilização desses animais, principalmente dos que possuem baixo valor genético, facilitando 0 manejo (COLAHAN et al., 1991). Este procedimento também está indicado nos casos de orquite, epididimite, neoplasia testicular, hidrocele, varicocele, traumas testiculares, torção do cordão espermático e hérnias inguinais (AUER; STICK, 2006). Previamente à cirurgia, os testículos devem ser palpados e avaliados para garantir que ambos estão na bolsa escrotal e que não há presença de hérnia inguinal (COLAHAN et al., 1991). O procedimento pode ser realizado com o animal em estação, sob efeito de sedação, ou em decúbito, sob efeito de anestesia geral (AUER; STICK, 2006; THOMAS et al., 1998).

Várias técnicas de castração têm sido desenvolvidas e aplicadas, cada uma com indicações e desvantagens que, o médico veterinário em conjunto com o proprietário, devem analisar, optando pelo procedimento mais adequado ao animal e às possibilidades econômicas (SOARES, 2009). A escolha da técnica é influenciada pela experiência pessoal do profissional (FINGER et al., 2011) e pelo comportamento animal, sendo que os cavalos que permitem palpação escrotal sem sedação são os principais candidatos à orquiectomia em posição quadrupedal (ADAMS; FESSLER, 2000; AUER; STICK, 2006).

As principais técnicas utilizadas para castração de equídeos são definidas como aberta, fechada e semifechada (AUER; STICK, 2006; SEARLE et al., 1999) e o acesso pode ser escrotal ou inguinal (AUER; STICK, 2006; SEDRISH; ACVS; LEONARD, 2001). A técnica aberta consiste na incisão completa da túnica vaginal e liberação do ligamento da cauda do epidídimo pela ruptura do mesórquio. Ligadura ou emasculação do plexo pampiniforme e ducto deferente são realizadas, seguida de remoção do testículo, epidídimo e porção distal do cordão espermático (AUER; STICK, 2006). Na técnica fechada não há abertura da túnica vaginal, com ligadura ou emasculação em bloco, da túnica vaginal, cordão espermático e plexo 
pampiniforme (AUER; STICK, 2006; FOSSUM, 2013). A técnica semifechada é semelhante à técnica fechada, porém há incisão da porção parietal da túnica vaginal. A emasculação das estruturas ocorre da mesma maneira que a técnica fechada (AUER; STICK, 2006).

Os cuidados no pós-operatório da orquiectomia incluem avaliação da presença de dor ou de complicações secundárias ao ato cirúrgico, como hemorragia e infecção. São indicadas a administração de analgésicos, a realização de curativos no local da cirurgia e a restrição da movimentação ou realização de exercícios controlados nos primeiros dias (FOSSUM, 2013). Segundo França (2005), os cavalos submetidos à orquiectomia eletiva, independente da técnica utilizada, são liberados para o trabalho em um prazo máximo de 30 dias.

Complicações pós orquiectomia podem ocorrer em curto ou longo prazo, sendo relatadas as hemorragias, edema escrotal, infecção incisional, peritonite, trauma peniano e eventração como principais ocorrências (THOMAS et al., 1998; THOMASSIAN, 2005). Com o intuito de minimizar essas complicações os veterinários devem estar cientes das principais ocorrências, reconhecer os fatores que predispõem seu desenvolvimento e serem capazes de resolvê-los (SCHUMACHER, 1996). O objetivo deste trabalho foi comparar 0 tempo cirúrgico, a ocorrência de complicações pós-operatórias e o tempo de internamento de cavalos submetidos à orquiectomia eletiva por meio de três diferentes técnicas abertas, empregadas por alunos e residentes do curso de medicina veterinária.

\section{MATERIAL E METODOS}

Neste trabalho foram selecionados 25 cavalos, de variadas raças e sem raça definida (SRD), com idade entre dois e 10 anos, sendo separados em três grupos, por randomização:

Grupo 1 (EMA): formado por oito cavalos, nos quais realizou-se orquiectomia bilateral, com incisão escrotal de aproximadamente $6 \mathrm{~cm}$, paralela à rafe mediana. A incisão estendeu-se às túnicas dartos e vaginal, até a exposição testicular. $\mathrm{O}$ mesórquio foi rompido com o auxílio manual ou de tesoura romba e uma pinça hemostática curva de $24 \mathrm{~cm}$ foi aplicada no segmento mais proximal do plexo vascular. $O$ cordão espermático foi ocluído com emasculador Reimer de 24 $\mathrm{cm}$, por três minutos para promoção de hemostasia da artéria e veias testiculares e o plexo foi seccionado um centímetro abaixo do local emasculado. A túnica vaginal foi mantida aberta, bem como a incisão, sendo o procedimento realizado com os cavalos em posição quadrupedal, com sedação e anestesia local.

Grupo 2 (BRA): formado por oito animais, nos quais foi realizada orquiectomia bilateral com a mesma técnica cirúrgica do grupo 1 , porém, o cordão espermático, foi ocluído com duas abraçadeiras de nylon de 2,5 milímetros de espessura, esterilizadas por autoclavagem. As braçadeiras foram colocadas na porção mais proximal do cordão espermático, com uma distância de um centímetro entre elas. O plexo foi seccionado um centímetro abaixo da segunda abraçadeira e a túnica vaginal foi mantida aberta, bem como a incisão, sendo o procedimento realizado com os cavalos em posição quadrupedal, com sedação e anestesia local.

Grupo 3 (ING): formado por nove animais, nos quais foi realizada orquiectomia bilateral com incisão inguinal, segundo técnica descrita por Sedrish et al. (2001) modificada. O testículo foi manualmente manipulado do escroto à região inguinal e exteriorizado por incisão de 
aproximadamente $6 \mathrm{~cm}$, incluindo na incisão as túnicas dartos e vaginal. $O$ mesórquio foi rompido com o auxílio dos dedos ou tesoura romba e uma pinça hemostática curva de $24 \mathrm{~cm}$ foi aplicada no segmento mais proximal do plexo vascular. $O$ cordão espermático foi ocluído com emasculador Reimer de 24 $\mathrm{cm}$, por três minutos, para promoção de hemostasia da artéria e veias testiculares e o plexo foi seccionado um centímetro abaixo do local emasculado. A túnica vaginal foi suturada com fio poliglactina $910 \quad 2-0$, em padrão contínuo simples e fixada à musculatura adjacente com um ponto simples. O espaço subcutâneo foi reduzido com sutura padrão Cushing e a dermorrafia foi realizada com sutura intradérmica, com fio poliglactina 910 2-0, em padrão contínuo simples. O procedimento foi realizado com os animais em decúbito dorsal, sob anestesia geral.

$$
\text { Para realização }
$$

dos

procedimentos os animais foram submetidos à jejum hídrico de seis e alimentar de 12 horas. Nos procedimentos realizados com os animais em estação, a sedação ocorreu por meio da administração intravenosa de $0,05 \mathrm{mg} \cdot \mathrm{kg}^{-1}$ de cloridrato de acepromazina, seguida de $1 \mathrm{mg} / \mathrm{kg}$ xilazina $10 \%$ associada a $0,036 \mathrm{mg} / \mathrm{kg}$ de butorfanol. Decorridos cinco minutos da sedação, era realizada aplicação intratesticular de ropivacaína $0,2 \%$, num volume de $20 \mathrm{~mL}$ por testículo, para bloqueio local.

Nas orquiectomias realizadas com os cavalos em decúbito dorsal, a medicação pré-anestésica foi realizada com acepromazina $1 \%(0,05 \mathrm{mg} / \mathrm{kg})$, por via intramuscular e 30 minutos após, era administrado xilazina $10 \%$, na dose de $0,5 \mathrm{mg} / \mathrm{kg}$ por via intravenosa. A indução ocorria pela administração intravenosa de guaifenesina $5 \%$ $(75 \mathrm{mg} / \mathrm{kg})$ associada ao propofol $(2 \mathrm{mg} / \mathrm{kg})$, a manutenção anestésica com isofluorano $1 \%$ e o bloqueio local com aplicação intratesticular de lidocaína $2 \%$, sendo administrado $20 \mathrm{~mL}$ em cada testículo.

Para todos os grupos, a analgesia pós-operatória foi realizada com flunixin meglumine na dose de 1,1 $\mathrm{mg} / \mathrm{kg}$ por via intravenosa durante três dias e antibioticoterapia com administração intramuscular de penicilina benzatina, na dose de 20000 $\mathrm{UI} / \mathrm{kg}$, totalizando três aplicações com intervalo de 24 horas entre elas.

O tempo dos procedimentos cirúrgicos nos diferentes grupos foram mensurados, sendo que nos grupos EMA e BRA o tempo considerado foi desde a incisão de pele até a secção do plexo vascular do segundo testículo e no grupo ING, o tempo considerado foi desde a incisão de pele até o término da sutura de pele.

Após todos os procedimentos, os animais foram observados durante 24 horas para verificar presença e grau de sangramento, estando os critérios utilizados nesta avaliação descritos na Tabela 1.

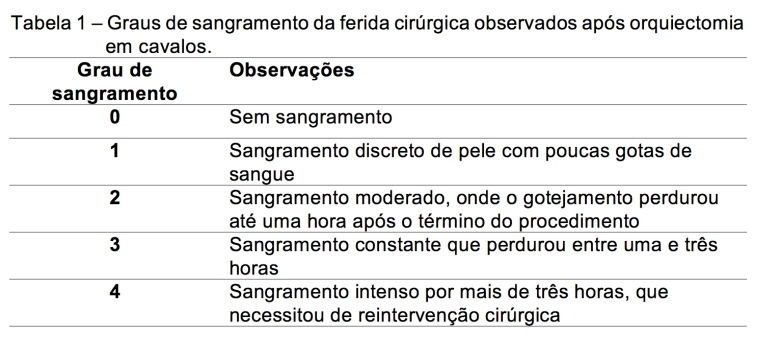

Durante 28 dias após o procedimento, em todos os animais foi avaliado grau de edema prepucial, presença de infecção local, manifestação de claudicação, tempo de internamento ou qualquer outra alteração física que o animal pudesse apresentar. As avaliações foram realizadas de forma direta, até mesmo nos animais que receberam alta antes de 28 dias.

Neste estudo, para análise estatística com comparação dos grupos, foi utilizada análise de variância One- 
way com comparação múltipla de Tukey.

\section{RESULTADOS E DISCUSSÃO}

O tempo médio em minutos e o desvio padrão dos procedimentos cirúrgicos foi de $16,3 \pm 2,5$ no grupo EMA, $14,5 \pm 1,6$ no grupo BRA e $64,4 \pm 16,6$ no grupo ING, como ilustrado no Gráfico 1.

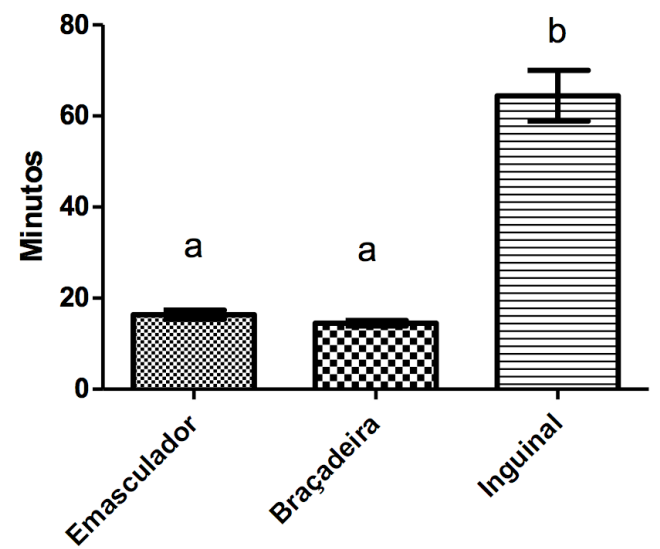

Gráfico 1 - Representação gráfica das médias de tempo de realização da cirurgia de orquiectomia utilizando diferentes técnicas.

Num estudo em que os autores compararam o tempo cirúrgico necessário para realização de orquiectomias com a utilização de diferentes materiais na hemostasia (abraçadeira de náilon, emasculador e categute), notou-se que, com a utilização da braçadeira de náilon o tempo de procedimento é reduzido (FRANÇA, 2005).

Sedrish et al. (2001), ao realizarem 35 orquiectomias em cavalos criptorquidas, através do acesso inguinal, obtiveram um tempo médio de procedimento de 35 minutos. O maior tempo de realização dos procedimentos neste trabalho, quando comparado ao tempo cirúrgico citado por Sedrish et al. (2001), pode estar relacionado à menor experiência dos alunos e residentes na realização da orquiectomia.
Segundo os critérios préestabelecidos para avaliação do grau de sangramento, todos os animais do grupo EMA apresentaram Grau 2 de sangramento, todos os animais do grupo BRA apresentaram Grau 1 e no grupo ING não foi observado sangramento pós-operatório em nenhum dos cavalos (Gráfico 2).

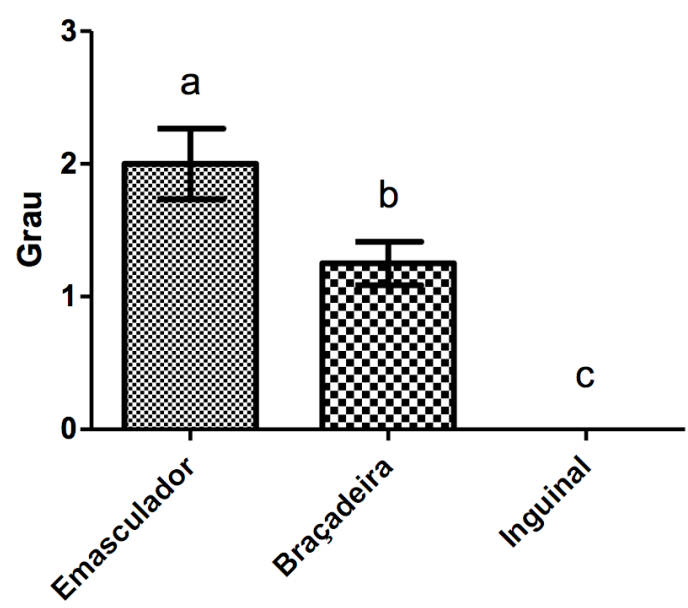

Gráfico 2 - Representação gráfica das médias de grau de sangramento após a realização da cirurgia de orquiectomia utilizando diferentes técnicas.

Sedrish et al. (2001) afirmam que há aumento na incidência de hemorragia pós-operatória nas orquiectomias realizadas com emasculadores quando comparadas às realizadas com ligaduras. A experiência que tivemos com este estudo corrobora com a afirmativa do autor, visto que os animais do grupo EMA apresentaram grau de sangramento maior do que os animais do grupo BRA. A hemorragia pós-operatória é comum nos primeiros 15 minutos e pode ser causada por utilização de emasculadores inapropriados ou por aplicação incorreta deste instrumento na castração, necessitando em alguns casos de reintervenção (SCHUMACHER, 1996). Neste trabalho o sangramento não perdurou por mais de 60 minutos, não necessitando maior atenção ou reintervenção cirúrgica. 
A presença de edema prepucial é comum após orquiectomias, sendo mais evidente no quarto ou quinto dia pósoperatório e quando excessiva, pode acarretar em contaminação local (SCHUMACHER, 1996). Exercícios controlados após 0 procedimento cirúrgico podem ser realizados para diminuir o edema local (SEDRISH; ACVS; LEONARD, 2001). Os grupos EMA e BRA apresentaram grau de edema semelhante entre si e muito maior do que o observado no grupo ING (Gráfico 3), no entanto não houve edema excessivo em nenhum animal deste estudo. Segundo Dart et al. (1999), a castração em decúbito dorsal é mais laboriosa do que a realizada em posição quadrupedal, porém, é menor a ocorrência de edema pós-operatório, e quando este ocorre, é menos grave. Sedrish et al. (2001) utilizaram no tratamento do edema pós-cirúrgico na orquiectomia a associação de antibióticos de amplo espectro, antinflamatórios não esteroidais, duchas de água fria e aumento de exercícios.

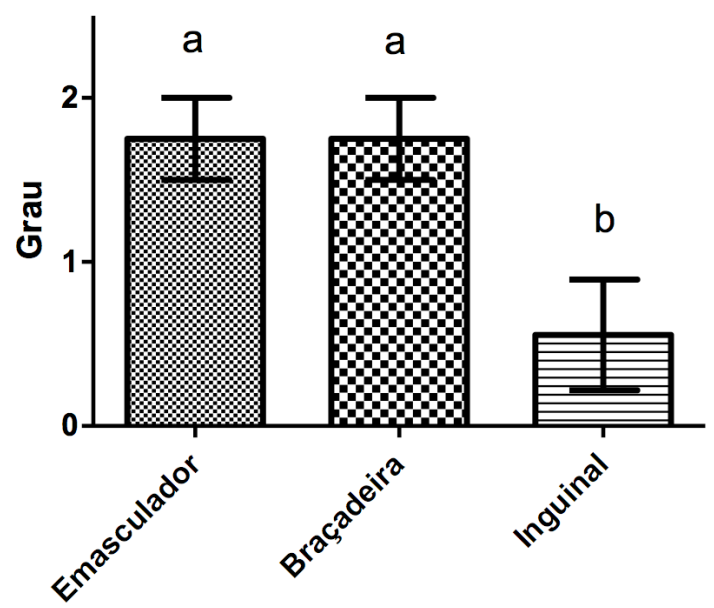

Gráfico 3 - Representação gráfica das médias de grau de edema de prepúcio após realização da cirurgia de orquiectomia utilizando diferentes técnicas.

Em todos os animais deste estudo foi realizada administração pósoperatória de antibióticos de amplo espectro e antinflamatório não esteroidal, independente da ocorrência ou ausência de edema. No entanto, nos animais que manifestaram aumento de volume pós-operatório, foram realizadas duchas, compressas com gelo e caminhadas supervisionadas até a redução do volume.

Nos cavalos do grupo EMA a alta

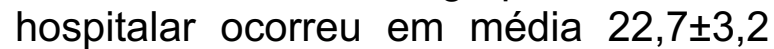
dias após o procedimento cirúrgico. Nos animais do grupo BRA ocorreu por volta de $20,75 \pm 0,7$ dias e no grupo ING esta média caiu para 10,7 $\pm 4,5$ dias (Gráfico 4). Para proprietários de cavalos de esporte, o tempo de internamento é um parâmetro importante no momento da tomada de decisão em relação à técnica de castração (SOARES, 2009). Segundo Dart et al. (1999), quando o animal é castrado sob anestesia geral com sutura da incisão cirúrgica o tempo de recuperação é menor, podendo este voltar às atividades normais entre sete e 10 dias depois do procedimento.

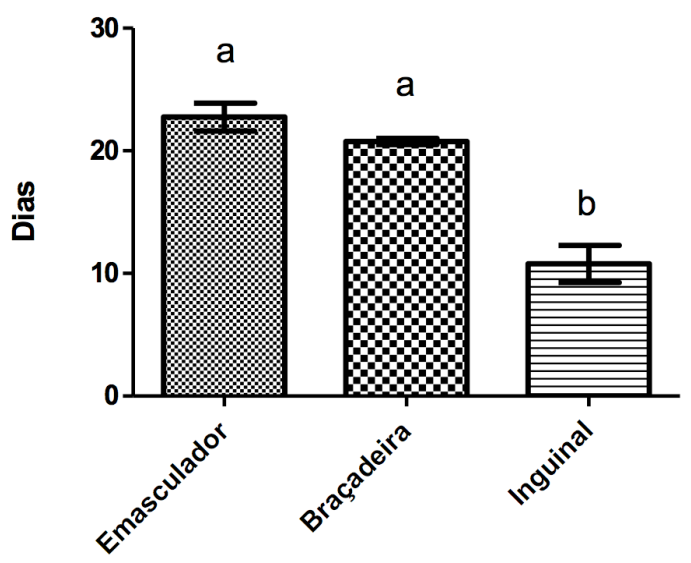

Gráfico 4 - Representação gráfica das médias de dias de internamento após a realização da cirurgia de orquiectomia utilizando diferentes técnicas.

Presença de secreção purulenta na ferida cirúrgica, caracterizando infecção local foi observada em dois animais do grupo EMA e em três do grupo BRA. No grupo ING não foi observada presença de secreção em nenhum dos cavalos operados. Segundo Sedrish et al. (2001), a técnica de orquiectomia com acesso inguinal e 
fechamento primário reduz a ocorrência de infecções incisionais. Nos animais que apresentarem infecção pósoperatória sugere-se utilizar doses elevadas de antibióticos, analgésicos e antinflamatórios não esteroidais, bem como remover as secreções e tecidos necrosados do local, realizando limpeza local (BRINSKO et al., 2003).

Outras complicações, que não foram verificadas em nenhum dos animais deste estudo, também podem ser encontradas no pós-operatório da orquiectomia, como evisceração, funiculite séptica, hidrocele, peritonite (SCHUMACHER, 1996) e febre (SEDRISH; ACVS; LEONARD, 2001)

Pode-se verificar nitidamente que no grupo ING o tempo de procedimento cirúrgico foi muito maior do que nos grupos EMA e BRA. Em contrapartida, nestes grupos houve sangramento e presença de edema no pós-operatório, os quais não foram observados no grupo ING. O tempo de internamento dos cavalos do grupo EMA e BRA foi mais que o dobro do tempo de internamento dos animais do grupo ING, ocasionando assim, um maior custo ao proprietário e um maior desgaste ao animal. Possivelmente, as complicações cirúrgicas, a dor causada por elas e a necessidade de cicatrização por segunda intenção elevaram o tempo de internamento dos animais nos grupos EMA e BRA. Sedrish et al. (2001), sugerem que a técnica inguinal com fechamento primário reduz as complicações pós-operatórias, o que condiz com o observado nos animais deste estudo.

\section{CONCLUSÃO}

A orquiectomia bilateral realizada sob efeito de anestesia geral e com fechamento da túnica vaginal, demanda um maior tempo cirúrgico quando comparada às orquiectomias realizadas com os cavalos em posição quadrupedal e manutenção da túnica aberta. Porém, não foram observadas complicações pós-operatórias como infecção, edema e hemorragia nos animais castrados com essa técnica, diminuindo assim o tempo de internação hospitalar.

\section{REFERENCIAS}

ADAMS, S. B.; FESSLER, J. F. Atlas of equine surgery. 1. ed. [s.l.] Saunders, 2000.

AUER, J. A.; STICK, J. A. Reproductive system. In: Equine Surgery. 3. ed. St. Louis, Missouri: Saunders, 2006. p. 775810.

BRINSKO, S. P. et al. Manual of equine reproduction. 3. ed. [s.l.] Elsevier Health Sciences Division, 2003.

COLAHAN, P. T. et al. Equine medicine and surgery. 4. ed. Goleta: American Veterinary Publications, 1991.

FINGER, M. A. et al. Comparação de duas técnicas de orquiectomia em equinos empregadas o ensino da técnica cirúrgica veterinária. Archives of Veterinary Science, v. 16, p. 53-59, 2011.

FOSSUM, T. W. Small Animal Surgery. 4. ed. St. Louis, Missouri: Elsevier Mosby, 2013.

FRANÇA, R. O. Ovariectomia e orquiectomia em equinos: uso da abraçadeira de náilon na hemostasia preventiva em comparação ao categute e emasculador. [s.l.] Universidade Federal de Goiás, 2005.

SCHUMACHER, J. Complications of castration. Equine Veterinary

Education, v. 8, n. 5, p. 254-259, 1996.

SEARLE, D. et al. Equine castration: review of anatomy, approaches, techniques and complications in normal, cryptorchid and monorchid horses. Australian veterinary journal, v. $77, \mathrm{n}$. 7, p. 428-434, 1999. 
SEDRISH, S. A.; ACVS, D.; LEONARD, J. M. How to Perform a Primary Closure Castration Using an Inguinal Incision. Animals, v. 47, p. 423-425, 2001.

SOARES, A. S. P. Estudo de uma técnica de castração de cavalos por laparoscopia. [s.l.] Universidade Técnica de Lisboa, 2009.

THOMAS, H. L. et al. Postcastration eventration in 18 horses: the prognostic indicators for long-term survival (19851995). Canadian Veterinary Journal, v. 39, n. 12, p. 764-768, 1998.

THOMASSIAN, A. Enfermidades dos

Cavalos. 4. ed. São Paulo: Varela, 2005. 